\title{
Revisión conceptual de los factores de riesgo psicosocial laboral y algunas herramientas utilizadas para su medición en Colombia*
}

\author{
A conceptual review of psychosocial, labor risk factors and \\ some instruments for measuring these factors in Colombia
}

\section{Revisão conceitual dos fatores de risco psicossociais no local de trabalho e algumas ferramentas usadas para sua medição na Colômbia}

\begin{abstract}
Andrés Lisímaco Polanco-Martínez
Magister en Administración de la Universidad del Valle, Especialista en Administración de la Calidad Total y la Productividad de la Universidad del Valle, Psicólogo de la Universidad Cooperativa de Colombia. Profesional contratista de la Secretaria de Paz y Convivencia del Municipio de Yumbo, Colombia.

andres.polanco@correounivalle.edu.co
\end{abstract}

\section{Mónica García-Solarte}

Ph. D Administración y Dirección de Empresas de la Universidad Politécnica de Cartagena - España. Master Of Management Tulane University, Magíster en Administración de Empresas de la Universidad del Valle. Profesora titular de la Universidad del Valle.

monica.garcia@correounivalle.edu.co.

Dirección de correspondencia: Calle 4B No.36-00. Edificio 124. Oficina 3013 Universidad del Valle Sede San Fernando, Cali - Colombia.

\section{Resumen}

Los factores de riesgo psicosocial laboral en Colombia, tienen un impacto significativo en la salud y la calidad de vida de los trabajadores que desarrollan actividades en su contexto de trabajo, haciéndose necesario su diagnóstico y la creación de programas de vigilancia epidemiológica de este factor que permitan prevenir accidentes de trabajo y enfermedades de origen laboral. Por lo anterior, en el presente documento se realiza un acercamiento conceptual desde la Organización Internacional del Trabajo - O.I.T., con diferentes autores y el marco legal en Colombia, que ayuda a evidenciar la importancia de la intervención de los factores de riesgo psicosocial laboral. Igualmente, se presentan algunas de las diferentes herramientas que se pueden emplear en Colombia para su medición y diagnóstico de acuerdo con los requerimientos de la Resolución 2646 del 2008 del Ministerio de la

\footnotetext{
* Cómo citar: Polanco-Martínez, A. L., García-Solarte, M. (2017). Revisión conceptual de los factores de riesgo psicosocial laboral y algunas herramientas utilizadas para su medición en Colombia. Revista Libre Empresa, 14(1), 111-131 http://dx.doi.org/10.18041/libemp.2017. v14n1.27105
} 
Protección Social, relacionados con el reconocimiento de tres tipos de condiciones de los factores de riesgo psicosocial laboral: intralaborales, extralaborales e individuales, en su evaluación. Lo anterior, permite identificar elementos que aporten a las organizaciones en la prevención de accidentes de trabajo y enfermedades de origen laboral.

\section{Palabras clave:}

Riesgo psicosocial laboral, prevalencia, estrés laboral.

\section{Clasificación JEL:}

D20, I10, L80, M12, M14, M52, M54.

\section{Abstract}

Psychosocial, labor risk factors in Colombia have a significant impact on the health and quality of life of workers who carry out activities in their workplace. Hence, it is necessary to diagnose and create epidemiological surveillance programs that make it possible to prevent accidents at work and occupational diseases. Therefore, this document accomplishes a conceptual introduction from the perspective of the ILO (International Labor Organization), various stakeholders, and the legal framework in Colombia that helps evidence the importance of conducting an intervention on psychosocial, labor risk factors. It also presents some of the different instruments that can be used in Colombia for measuring and diagnosing these risk factors in compliance with the requirements in Resolution 2646 of 2008 issued by the Ministry for Social Protection with regard to the acknowledgment of three kinds of conditions of psychosocial, labor risk factors in their assessment, namely, inside the workplace, outside the workplace, and individual factors. This makes it possible to identify aspects that assist organizations with the prevention of accidents at work and occupational diseases.

\section{Key words}

Psychosocial, labor risk, prevalence, work stress.

\section{Resumo}

Os fatores de risco psicossocial laboral na Colômbia têm um impacto significativo na saúde e qualidade de vida dos trabalhadores que desenvolvam atividades no seu contexto de trabalho, tornando-se necessário o seu diagnóstico e a criação de programas de vigilância epidemiológica desse fator que permitam prevenir acidentes e doenças relacionadas ao trabalho. Por isso, neste documento é realizada uma abordagem conceitual pela Organização Internacional do Trabalho (OIT), diferentes autores e enquadramento legal na Colômbia, que ajuda a destacar a importância da intervenção dos fatores de risco psicossocial laboral. Igualmente, são apresentadas algumas das diferentes ferramentas que podem ser usadas na Colômbia para sua medição e diagnóstico de acordo com as exigências da Resolução 2646 de 2008 do Ministério de Proteção Social, relacionados com o reconhecimento de três tipos de condições dos fatores de risco psicossocial laboral: intra laborais, extra laborais e individuais, em sua avaliação. $\mathrm{O}$ anterior permite identificar os elementos que contribuem 
para as organizações na prevenção de acidentes de trabalho e doenças relacionadas ao trabalho.

\section{Palavras chave}

Risco psicossocial laboral, prevalência, estresse laboral.

\section{Introducción}

En el momento de realizar una revisión conceptual acerca de los factores de riesgo psicosociales laborales, se logra identificar que no existe una unificación sobre su conceptualización. Por lo cual, podemos decir inicialmente que estos factores según Álvarez, Conti, Valderrama, Moreno, y Jiménez (2007) son aquellas condiciones presentes en un escenario laboral y que se encuentran inmersas en la organización, el contenido del trabajo y la ejecución de las tareas, que perturban el bienestar o la salud física, psíquica y social del trabajador, así como la misma gestión laboral. Por lo tanto, los factores de riesgo psicosocial laboral “(...), son aquellas características de las condiciones de trabajo y, sobre todo, de su organización que afectan la salud de las personas a través de mecanismos psicológicos y fisiológicos" (Gamero, 2013, p.210).

De esta forma, la presencia de estos factores en las empresas se genera según Mateo (2013) cuando los factores psicosociales de la organización no son adecuados y, la consecuencia de esto puede provocar respuestas fisiológicas de estrés, que pasan a ser factores psicosociales de riesgo o estresores. Se puede afirmar que se trata de factores de la organización que logran representar riesgo de afectar negativamente la salud del trabajador. Teniendo en cuenta lo anterior, autores como Varilla (2009) refieren que no se pueden desconocer las consecuencias de la acción nociva de los factores psicosociales para el bienestar de los trabajadores, y que estas no se limitan a la vivencia de una tensión emocional molesta y pueden llegar a convertirse en efectos más profundos.

Ahora bien, en Colombia a partir de la expedición de la resolución 2646 del año 2008 por parte del Ministerio de la Protección Social, se establecen las directrices para su intervención, también se definen los responsables de su realización, se conceptualiza, entre otros elementos de los factores de riesgo psicosocial laboral para un mejor acercamiento y diagnóstico de las situaciones o condiciones generadoras de estos factores dentro de las empresas colombianas. Por lo tanto, al estudiar los factores de riesgo psicosociales en el trabajo se hace necesario la identificación de los diferentes elementos que conforman su concepto, el desarrollo de diversos métodos de intervención y diagnóstico utilizados en Colombia por las empresas, que permiten reconocer las actuaciones de las empresas hacia los empleados y los efectos positivos que contribuyen a la salud en el trabajo.

En una primera aproximación se realiza una breve descripción teórica sobre los factores de riesgo psicosocial laboral, que permite identificar los elementos que constituyen su concepto y su relación con su estudio en las empresas, posteriormente se abordan los elementos claves para la intervención mediante las herramientas validadas en el contexto 
para el cumplimiento de las exigencias de la legislación colombiana, que genere aportes a los trabajadores y a las empresas, en el propósito de la identificación de los factores y la realización de acciones que conlleven a la disminución y eliminación de las enfermedades de origen laboral.

\section{Los factores de riesgo psicosociales en el trabajo}

Los factores de riesgo psicosociales laborales pueden reconocerse como una condición o situación que enfrenta un trabajador en su contexto laboral, y cuya exposición puede afectar de manera negativa su salud a nivel mental, físico y social, teniendo en cuenta los diferentes aspectos relacionados con las características personales del sujeto, en las cuales se involucra el ambiente laboral, familiar y social. Se puede identificar que estos factores de riesgo comprenden aspectos intra-laborales, extra-laborales o externos a las empresas, sin dejar de lado aquellas características inherentes del trabajador, que mediante la interacción de percepciones y experiencias, inciden en la salud y el desempeño de las personas en el contexto laboral (Ministerio de Trabajo y Seguridad Social [MTySS], Ley 2646 de 2008).

Por su parte, la Organización Internacional del Trabajo - O.I.T., define estos factores de riesgo como:

Interacciones entre el trabajo, su medio ambiente, la satisfacción en el trabajo y las condiciones de su organización, por una parte, y por la otra, las capacidades del trabajador, sus necesidades, su cultura y su situación personal fuera del trabajo, todo lo cual, a través de percepciones y experiencias, puede influir en la salud y en el rendimiento y la satisfacción en el trabajo (Comité Mixto O.I.T. y O.M.S, 1984. p.12).

Mientras que la Enciclopedia sobre la Seguridad y Salud en el Trabajo, según Sauter, Murphy, Hurrel \& Levi (2001) define los factores de riesgo psicosocial laboral como:

(...), aspectos del puesto de trabajo y del entorno de trabajo, como el clima o cultura de la organización, las funciones laborales, las relaciones interpersonales en el trabajo y el diseño y contenido de las tareas (por ejemplo, su variedad, significado, alcance y carácter repetitivo). El concepto de factores psicosociales se extiende también al entorno existente fuera de la organización (por ejemplo, la doble presencia trabajohogar) y aspectos del individuo (por ejemplo, personalidad y actitudes) que puedan influir en la aparición del estrés en el trabajo. (p.34.2)

Los factores psicosociales laborales se han conceptualizado por parte de varios autores, lo que ha conllevado que a la fecha no exista una unificación conceptual respecto a este factor. Siendo así, se expondrán algunos de estos conceptos con el fin de tener una aproximación frente a este tema. Por lo tanto, Varillas (2009) refiere que los factores psicosociales son condiciones que propician el estrés laboral y también conllevan otras complicaciones que relacionan la salud y la seguridad. 
Por otra parte, pueden considerarse estos factores como significativos dentro de las organizaciones y cuyos efectos tienen incidencia directamente en la salud de los empleados, por lo cual Creus (2011) refiere que los factores de riesgo psicosociales comprenden un conjunto de demandas, también la singularidad del trabajo y su organización, y cuando esto coincide con las capacidades, exigencias y expectativas del trabajador, tienen incidencia en su salud.

No se puede dejar de lado que otro de los efectos de los factores de riesgo psicosociales en el trabajo, según Creus (2011) hace referencia a la existencia de un desequilibrio en la salud del trabajador, como efecto de la dificultad de dar una respuesta apropiada a las exigencias del trabajo, dado que observa frenadas sus aspiraciones y expectativas, y esto recibe el nombre de estrés. En otras palabras, autores como González Giachero, y Delgado (2012) refieren que se pueden definir genéricamente estos riesgos, como las condiciones existentes en el ámbito laboral, y que se relacionan con la organización del trabajo, el contenido del trabajo y la realización de las tareas, las cuales tienen la capacidad de afectar el desarrollo del trabajo y la salud del trabajador.

Por su parte, Mateo (2013) refiere que los factores de riesgo psicosociales hacen referencia a las condiciones de la organización y que estas son continuas en la empresa. Por lo tanto, son todos aquellos factores derivados de cómo se desarrolla la gestión de las empresas. En cambio, Gamero (2013) manifiesta que estos factores de riesgo se refieren a las condiciones presentes en un escenario laboral y se encuentran directamente vinculados con la organización, el volumen del trabajo, el clima laboral y la ejecución de la tarea, y poseen la suficiencia de afectar tanto el bienestar o la salud biopsicosocial del trabajador como la realización del trabajo.

Al respecto, autores como Vieco, y Abello (2014) refieren que los factores de riesgo psicosociales laborales, poseen propiedades relacionadas con las condiciones del trabajo, y de su organización, las cuales posiblemente afectan la salud de los individuos mediante elementos psicológicos y fisiológicos, que igualmente se les denomina estrés. No obstante, los factores de riesgo psicosocial, según Bestratén (2015) refieren que pueden conceptualizarse como los originados por los aspectos organizativos del trabajo, y como tal estos provocan en el trabajador insatisfacción laboral, estrés y otros problemas que repercuten en la cantidad y la calidad de sus labores.

Por consiguiente, los conceptos expresan como elemento común que la organización es la fuente de los factores de riesgo psicosocial laboral, tanto en el ejercicio de la dinámica administrativa, como de su operación, y que las características individuales de los empleados juegan un papel importante respecto a la exposición a este factor, lo cual conllevará afectos negativos en la salud y puede convertirse en una causa de accidentes laborales y de las enfermedades de origen laboral como el estrés.

Por lo tanto, es importante reconocer la definición del estrés como manifestación de los factores psicosociales laborales, la Universidad Pontificia Javeriana, en el Subcentro de Seguridad Social y Riesgos Profesionales (2007) refiere que el estrés es la agrupación 
de respuestas psicológicas, emocionales y comportamentales, que se producen en el momento que el individuo debe hacer frente a las exigencias que se derivan de su dinámica con el escenario en el cual se encuentra expuesto a los factores de riesgo psicosociales, donde su capacidad de afrontamiento no alcanza a suplir las demandas de este escenario de riesgos, por lo tanto se genera un desbalance que altera su bienestar e incluso su salud.

En cambio, el estrés laboral, de acuerdo con la Enciclopedia sobre la Seguridad y Salud en el Trabajo, según Levi, L. (2001) se define como:

La terminología técnica, la tensión (inglés Stress) es "una fuerza que deforma los cuerpos”. En biología y medicina suele utilizarse el término estrés para denominar un proceso corporal, el plan general del cuerpo para adaptarse a todas las influencias, cambios, exigencias y tensiones a que está expuesto. (Cap.34.3)

Teniendo en cuenta otras perspectivas, la conceptualización del estrés laboral según Barrios Baudor - Oros Muruzabal-Rivas Vallejo (Citado por Rivas, 2009) afirman que: “(...), constituye una forma patológica de reaccionar frente a los estímulos y a la tensión extrema, sobre cuyas causas no existe consenso, aunque se apunta a un posible trastorno genético en la bioquímica del cortisol” (p.7).

Por su parte, Moreno y Garrosa (2013) refieren que el estrés puede entenderse como un estímulo, cuando una fuerza es aplicada a una persona y lo que le produce esto es una determinada alteración. Sin embargo, respecto al estrés López (1996) expresa que se genera, debido al desequilibrio entre las exigencias y las condiciones del medio de trabajo, sin dejar de lado las necesidades, las facultades y expectativas individuales del trabajador. Siendo así, Arias (2012) anota que el estrés presenta efectos negativos sobre la salud en el momento en que es intenso y prolongado. Siendo más claro, cuando sobrepasa las herramientas individuales de la persona para poder enfrentarlo.

Por lo tanto, se logra evidenciar que hasta el momento no se presenta una unificación teórica respecto al concepto del estrés. Por lo tanto, Moreno, y Garrosa (2013) afirman que este fenómeno es cada vez más frecuente en el contexto y el mundo del trabajo. Siendo así, existen diferentes propuestas respecto a su definición. De acuerdo con lo anterior, Boada y Ficapal (2012) presentan algunas definiciones, que se exponen en la Tabla 1.

\section{Tabla 1}

Algunas definiciones del estrés

\begin{tabular}{|l|l|}
\hline \multicolumn{1}{|c|}{ Autores } & \multicolumn{1}{c|}{ Definiciones } \\
\hline $\begin{array}{l}\text { Ruff y Korchin } \\
\text { (en 1967) }\end{array}$ & $\begin{array}{l}\text { Un esfuerzo agotador para mantener las funciones esenciales } \\
\text { al nivel requerido. }\end{array}$ \\
\hline Banner (en 1967) & Una frustración y amenaza que no pueden reducirse. \\
\hline
\end{tabular}




\begin{tabular}{|l|l|}
\hline Levi (en 1971) & $\begin{array}{l}\text { Una información que el sujeto interpreta como amenaza de } \\
\text { peligro. }\end{array}$ \\
\hline $\begin{array}{l}\text { Groen y Bastiaans } \\
\text { (en 1975) }\end{array}$ & Una imposibilidad de predecir el futuro. \\
\hline $\begin{array}{l}\text { Lazarus y Folkman } \\
\text { (en 1984) }\end{array}$ & $\begin{array}{l}\text { Es la relación entre la persona y el ambiente que es apreciado } \\
\text { por la persona como gravoso o con demandas superiores a } \\
\text { sus recursos y dañino para su bienestar. }\end{array}$ \\
\hline $\begin{array}{l}\text { Valdés y De Flores } \\
\text { (en el 2000) }\end{array}$ & $\begin{array}{l}\text { Un estado que se sitúa entrela sobreestimulación o sobrecarga } \\
\text { y la acción atenuadora o restauradora del organismo. }\end{array}$ \\
\hline $\begin{array}{l}\text { Coon } \\
\text { (en el 2004) }\end{array}$ & $\begin{array}{l}\text { Es la condición mental o física que ocurre en cualquier } \\
\text { momento en el que debemos ajustarnos o adaptarnos al } \\
\text { ambiente. }\end{array}$ \\
\hline $\begin{array}{l}\text { Rosenzweig } \\
\text { (en el 2005) }\end{array}$ & $\begin{array}{l}\text { Es la situación-estímulo que constituye un obstáculo o una } \\
\text { obstrucción más o menos insuperable en la vía que conduce } \\
\text { a la satisfacción de una necesidad vital cualquiera. }\end{array}$ \\
\hline
\end{tabular}

Fuente: (Boada, y Ficapal, 2012, p.106)

\section{Factores de riesgo psicosocial laboral en Colombia}

La contextualización de los factores de riesgo psicosociales en Colombia, teniendo como referente la resolución 2646 de 2008 del Ministerio de la Protección Social, se encuentra encaminada hacia el reconocimiento conceptual de este factor, las normas asociadas y la generación de acciones para disminuir la ocurrencia de accidentes en el contexto del trabajo y la adquisición de enfermedades con origen laboral. En este sentido, Gil (2012) refiere que el origen del problema en las situaciones de riesgo psicosocial no se encuentra en el trabajador, sino que se producen en el contexto donde se generan las situaciones de riesgo, dado el inadecuado diseño y organización del trabajo.

Teniendo en cuenta lo anterior, en Colombia se expide por parte del Ministerio de Trabajo y Seguridad Social, el Decreto 614 (1984) que determina los elementos y bases para la organización y administración en lo concerniente a la Salud Ocupacional. Así, de acuerdo con López (2015): "Sería entonces a través de un decreto reglamentario expedido por el ejecutivo como se abriría el camino por el cual el riesgo psicosocial ingresaría a la legislación colombiana” (p.64). Sin dejar de lado, otros aspectos también significativos como el reconocimiento de la Salud Ocupacional, la identificación de los factores de riesgo y la necesidad de tomar medidas preventivas para la minimización de enfermedades laborales y accidentes dentro del contexto laboral.

Posteriormente, tras la expedición del decreto 1295 del año 1994, se establecen, entre algunos objetivos, las actividades de promoción y prevención en busca de la mejora de las condiciones de trabajo y la salud de los trabajadores (Ministerio de Trabajo y Seguridad Social [MTySS], Decreto 1295 de 1994). Años más tarde, se expide la Ley 1010 del 2006, la cual define y/o tipifica los comportamientos que se consideran acoso 
laboral y establece las acciones para prevenir, corregir y sancionar el acoso laboral en lo comprendido en las relaciones que se presentan dentro del escenario del trabajo (Congreso de Colombia Ley 1010, 2006). Concibiéndose como una herramienta para exponer ante las entidades encargadas de regular las empresas públicas y privadas, las situaciones y comportamientos a los cuales se enfrentan los trabajadores en el desarrollo de sus actividades laborales.

En el año 2008, se conceptualiza en Colombia el factor de riesgo psicosocial laboral mediante la emisión de la Resolución 2646 (2008), la cual refiere las disposiciones y definición de los responsables en lo que concierne a la determinación, valoración, actuación y seguimiento continuo de la exposición a los factores de riesgo psicosociales en el trabajo y también contribuye a la identificación del origen de las enfermedades ocasionadas por el estrés laboral.

En cumplimiento de esta normatividad las empresas deben iniciar el proceso de intervención de este factor, estableciendo un programa enfocado a la promoción y prevención del riesgo psicosocial, empleando un método de evaluación acorde con las necesidades de la población trabajadora y de esta forma desarrollar acciones correctivas, preventivas y de mejoramiento, con el fin de minimizar sus efectos negativos en la salud de los empleados. Teniendo en cuenta lo anterior, Tomasina (2012) refiere que el proceso salud-enfermedad se halla determinado, en la mayoría de los casos, por los espacios y contextos laborales donde los trabajadores se encuentran inmersos.

Luego, con la expedición de la Ley 1562 del 2012, se transforma el sistema de riesgos laborales y se enmarcan otras condiciones para la Salud Ocupacional en Colombia, abordando una nueva conceptualización para la salud ocupacional, que mediante esta Ley se entenderá como seguridad y salud en el trabajo, sin dejar de lado que la define como aquella disciplina que trata de la prevención de la protección y promoción de la salud de los trabajadores (Ministerio de Trabajo y Seguridad Social [MTySS], Ley 1562 de 2012).

Es de aclarar que a las organizaciones, sus empleadores; representantes y personal relacionado con el desarrollo de actividades de prevención de incidentes y promoción de la salud en el trabajo, se les hace indispensable conocer y comprender la conceptualización de accidente de trabajo. Donde este concepto hace referencia a todo evento que se desarrolle a causa del trabajo, y que como tal, genere algún tipo de lesión orgánica, perturbación funcional, psiquiátrica, una invalidez o la muerte de este trabajador (Ministerio de Trabajo y Seguridad Social [MTySS], Ley 1562, 2012).

Cabe notar que estos sucesos pueden conllevar hacia la adquisición de enfermedades laborales, que afectan directamente al trabajador. Se debe reconocer entonces que una enfermedad ocupacional es la adquirida como consecuencia de la exposición a diferentes factores de riesgo presentes en las actividades laborales o en el escenario donde el trabajador ejecuta tales tareas (Ministerio de Trabajo y Seguridad Social [MTySS], Ley 1562, 2012).

Con el reconocimiento de estos conceptos y las respectivas sensibilizaciones a los trabajadores mediante los planes de capacitación de las empresas, se abordarán 
actividades del orden de la prevención que tengan un enfoque de autocuidado, en aras de la buena salud en el contexto laboral. Posteriormente, se expide el Decreto 1443 del 2014, por el cual se expresan las disposiciones para implementar el sistema de Gestión de la Seguridad y Salud en el Trabajo (Ministerio de Trabajo, Decreto 1443 de 2014). Pasando de un simple programa, a un sistema que se puede articular con otros sistemas de gestión en la empresa, que permitan mejorar la eficiencia administrativa y trabajar hacia la promoción y prevención de la salud dentro de los entornos laborales.

Luego, mediante el Decreto 1072 del año 2015 se definen los lineamientos de obligatorio cumplimiento hacia la implementación del Sistema de Gestión de la Seguridad y Salud en el Trabajo, cuya obligación corresponde a todas las empresas colombianas y aplicadas a todo tipo de trabajadores en el contexto colombiano (Ministerio del Trabajo, Decreto 1072, 2015).

Esta revisión normativa, permite reconocer que en Colombia han existido cambios progresivos en relación con el tema de Salud Ocupacional, hoy denominado Seguridad y Salud en el Trabajo, y en lo que respecta a la identificación de factores de riesgo psicosociales laborales, donde se evidencia la necesidad de trabajar a nivel de la prevención y el desarrollo de actividades que permitan promover la identificación temprana de los factores de riesgo y la realización de planes correctivos y de mejoramiento, al presentarse la existencia de estos factores.

Los principales cambios de la Seguridad y Salud en el Trabajo en Colombia se han desarrollado de acuerdo con las necesidades de adaptación hacia sistemas de gestión, los cuales permiten la articulación con otros sistemas y ser más eficientes en el desarrollo de actividades dirigidas hacia la prevención de los riesgos, la accidentalidad y las enfermedades de origen laboral. Así mismo, la normatividad incide en que las empresas tienen que medir las condiciones intralaborales, extralaborales y las condiciones individuales, con miras a la identificación de los factores de riesgo psicosocial laboral.

De acuerdo con lo anterior, se hace necesario el reconocimiento conceptual de las condiciones que se exigen respecto a la valoración de los factores de riesgo psicosocial laboral. Por lo tanto, el Ministerio de la Protección Social define estas condiciones, para el caso de las condiciones intralabores,

(...), son entendidas como aquellas características del trabajo de su organización que influyen en la salud y bienestar del individuo" (Ministerio de la Protección Social, 2010, p.20). Respecto a las condiciones extralaborales, estas "comprenden los aspectos del entorno familiar, social y económico del trabajador. A su vez, abarcan las condiciones del lugar de vivienda, que pueden influir en la salud y bienestar del individuo" (Ministerio de la Protección Social, 2010, p.26).

Sin dejar de lado que las condiciones individuales, según el Ministerio de la Protección Social (2010) refiere que las propiedades de cada trabajador o particularidades socio-demográficas como el sexo, la edad, estado civil, nivel educativo, la ocupación 
(entendida como profesión u oficio), la ciudad de domicilio, el estrato socioeconómico, las características de la vivienda, y la cantidad de personas que dependen del trabajador. Estas características pueden influir en la percepción y en el efecto de los riesgos intralaborales y extralaborales.

\section{Herramientas diseñadas para la medición del factor de riesgo psicosocial laboral en Colombia}

Autores como Bocanument, y Barján en 1993, crean un instrumento para la medición de los factores de riesgo psicosocial, en aras de contar con una herramienta que facilitara la identificación de estos factores en las empresas colombianas. De acuerdo con lo anterior, Charria, Sarsosa, y Arenas (2011) refieren que en Colombia son pocos los instrumentos diseñados con las particularidades de la población trabajadora, que faciliten la evaluación de los factores de riesgo psicosociales en las empresas.

Sin embargo, mediante la expedición de la Resolución 2646 de 2008, emitida por el Ministerio de la Protección Social, abre un camino normativo hacia el cumplimiento de la intervención de los factores de riesgo psicosocial laboral para las empresas en Colombia. Gómez y Moreno (2010) refieren que existen retos para esta resolución, y uno de ellos es validar en el entorno colombiano los modelos y herramientas ya aprobados en otros países. También expresan la necesidad de construir mapas de factores de riesgo, teniendo en cuenta las ocupaciones, los sectores económicos, los géneros, etc., de este país.

Es decir, Colombia presentaba la necesidad de una herramienta que reconociera las variables requeridas y las condiciones exigidas por la Resolución 2646 de 2008, para la medición del factor de riesgo psicosocial y que estuviera validada con su población trabajadora y en empresas colombianas. Por lo tanto, el Ministerio de la Protección Social de Colombia, en el año 2010 presenta la batería de instrumentos que valora los factores de riesgo psicosocial, la cual se encuentra validada en el contexto colombiano, esto con el fin de ofrecerle a las empresas públicas y privadas, iniciar con la identificación y evaluación de los factores de riesgo psicosocial intra y extralaboral, así como el desarrollo de acciones para la prevención de accidentes de trabajo y enfermedades de origen laboral. Es de resaltar que la utilización de este instrumento no tiene costo alguno.

Por lo tanto, es pertinente tener en cuenta autores como Bestratén (2015) el cual refiere que la evaluación de los riesgos psicosociales requiere de la aplicación de cuestionarios expresamente concebidos para tomar la información y las opiniones de los trabajadores que se encuentran enfrentando la exposición a estos factores de riesgo, donde es importante que se reconozca la percepción de todos los trabajadores y no se implementen técnicas de muestreo en el momento de realizar la evaluación. De esta forma, el Ministerio de Trabajo y Seguridad Social, en la Ley 2646 (2008) refiere que la valoración de los factores psicosociales y sus efectos, debe realizarse a través de instrumentos que para el efecto hayan sido validados en Colombia.

Siendo así, no se puede dejar de lado que en las actuaciones hacia los factores de riesgo psicosociales deben involucrarse los directamente interesados o afectados (Ministerio de 
Trabajo y Seguridad social [MTySS], Ley 2646, 2008). De acuerdo con lo anterior, Varillas (2009) refiere que para la medición del factor de riesgo psicosocial, se debe contar con una metodología que conlleve la realización de un protocolo para desarrollar el proceso de medición.

En este orden de ideas, la Resolución 2646 (2008) refiere que la evaluación de estos factores debe realizarse anualmente por parte de las empresas, teniendo en cuenta que deben confrontarse los objetivos inicialmente planteados con los logros que se obtuvieron de la medición. Siendo así, las condiciones con las cuales se realiza la medición de los factores de riesgos psicosociales laborales, corresponde a las exigencias de la resolución 2646 del 2008 y los principios metodológicos deben brindarlos cada una de las herramientas o instrumentos elaborados para la medición del factor del riesgo psicosocial laboral.

Cabe destacar que la batería del Ministerio de la Protección Social se reconoce como instrumento validado con las características de los sectores económicos y trabajadores colombianos, dado que del Instrumento de Bocanument y Barján no se encuentran evidencias de su validación y su diseño se presentó antes de emitirse la resolución 2646 de 2008 que brinda las directrices para la medición del factor de riesgo psicosocial en Colombia. Por lo tanto, mediante la utilización de una batería validada estadísticamente con la población trabajadora y empresas colombianas, de acuerdo con los sectores económicos de este país, se obtendrá mayor confianza en sus resultados, lo cual permitirá la toma objetiva de acciones acordes con las necesidades de los trabajadores y de cada una de las empresas.

Mateo (2013) afirma que realizar el tratamiento de los riesgos psicosociales con enfoque preventivo hace referencia a seguir una secuencia de acciones, tales como: la identificación del riesgo, la evaluación cuando no se haya podido evitar, la intervención para eliminarlo o reducirlo y la realización del respectivo seguimiento para medir la eficacia de la intervención.

Teniendo en cuenta lo anterior, Buceta y Bueno (2001) refieren que encontrándose la presencia del estrés en la organización, la intervención no debe limitarse a eliminar o aliviar sus manifestaciones, también debe dirigirse a debilitar las condiciones del contexto y las personales que lo favorecen. Esto en la búsqueda del desarrollo de características personales eficaces y saludables, que ayuden a mejorar los logros de la intervención.

Por otra parte, se hace necesario reconocer la importancia del porqué de la evaluación de los factores de riesgo psicosociales laborales en las empresas, donde no se puede dejar de lado que esta medición está inmersa dentro de un Sistema de Seguridad y Salud en el Trabajo enmarcado en la normatividad colombiana mediante el Decreto 1072 del 2015, el cual es de obligatorio cumplimiento para las empresas. Este sistema en las empresas debe poseer lineamientos claros y metas definidas para el mejoramiento de la calidad de vida de los trabajadores. Por lo tanto, Henao (2013) estima que la seguridad y la salud en el trabajo tiene que direccionarse hacia el cumplimiento de sus políticas, estrategias, acciones y recursos, con lo cual obtendrá el mejoramiento de la calidad de vida y salud 
de la población trabajadora, además de ser un instrumento para la mejora en la calidad, productividad y eficiencia de las empresas, incluso el mejoramiento de la sociedad.

Actuaciones que deben ser llevadas a cabo por la empresa, la gerencia o quien desempeñe el rol de representante de la organización. Autores como Peiró (2009) refieren que el empleador se encuentra en la obligación de intervenir los riesgos laborales, incluidos los psicosociales. Siendo así, las empresas públicas y privadas de Colombia, deben asumir la responsabilidad y las consecuencias del incumplimiento de la normatividad que regula la identificación, evaluación, intervención y monitoreo permanente a los factores de riesgo psicosociales laborales, que conlleven al reconocimiento del origen de las enfermedades generadas por el estrés ocupacional (Ministerio de Trabajo y Seguridad social [MTySS], Ley 2646 de 2008). Por lo tanto, el Capítulo V de la Resolución 2646, emitida por el Ministerio de la Protección Social (2008) afirma:

El incumplimiento a lo establecido en la presente resolución será sancionado, de conformidad con lo dispuesto en los literales a) y c) del artículo 91 del Decreto-ley 1295 de 1994. La investigación administrativa y la sanción serán de competencia de las Direcciones Territoriales del Ministerio de la Protección Social, de conformidad con lo previsto en el artículo 115 del Decreto-ley 2150 de 1995. (p.9)

De acuerdo con lo anterior, el Decreto 1295 de 1994 expedido por el Ministerio de Gobierno de la Republica de Colombia, expresa que:

Cuando el empleador o responsable del pago de la cotización no aplique las instrucciones, reglamentos y determinaciones de prevención de riesgos profesionales, adaptados en forma general por la Dirección Técnica de Riesgos Profesionales del Ministerio de Trabajo y Seguridad Social, esta le podrá imponer multas mensuales consecutivas hasta por quinientos (500) salarios mínimos legales mensuales (p.28).

Vale la pena decir que la renuencia grave al incumplimiento de esta norma, y cuando los riesgos profesionales generados por la empresa así lo ameriten, el Decreto 1295 (1994) refiere que la Dirección Técnica de Riesgos Profesionales del Ministerio de Trabajo y Seguridad Social, tiene la libertad de ordenar la suspensión de actividades de las empresas. En efecto, Colombia ha establecido directrices, instrucciones y recomendaciones para llevar a cabo las medidas de prevención frente a los riesgos psicosociales laborales y la promoción de la salud en el contexto laboral, para lo cual las empresas públicas y privadas se encuentran inmersas en estas exigencias, y deberán desarrollar un Sistema de Seguridad y Salud en el trabajo enfocado hacia la prevención de accidentes de trabajo y enfermedades de origen laboral en los empleados, de acuerdo con las exigencias de ley.

\section{Instrumento de Guillermo Bocanument Zuluaga y Norby Piedad Barján Bahamón para la identificación de factores de riesgo psicosociales}

Bocamument (1994) refiere que el instrumento hizo parte del trabajo de grado presentado por la enfermera Norby Piedad Barján Bahamón, para optar al título de especialista en Salud Ocupacional de la Facultad Nacional de Salud Pública de la Universidad de Antioquia, donde su rol fue como asesora de esta tesis. 
De acuerdo con Bocanument (1994) en el año 1993 se diseñó un instrumento coherente, versátil y operativo que permitirá hacer lectura objetiva de la realidad de los factores de riesgo psicosociales; la encuesta construida los agrupa en cinco áreas fundamentales: Contenido de tarea, relaciones humanas, organización del tiempo de trabajo y gestión de personal, en la quinta área se exploran las alteraciones físicas y psíquicas relacionadas con situaciones estresantes del trabajo. Este instrumento posee en totalidad 85 preguntas, cada una de ellas contiene una valoración específica, y el evaluado debe seleccionar de acuerdo con su percepción, en una escala que contiene los siguientes criterios: casi siempre, la gran mayoría, algunas veces y casi nunca.

Por lo cual, Bocanument (1994) afirma:

Los resultados de la aplicación permiten obtener grados de peligrosidad (alto, medio y bajo), bien en forma global o parcializados por área. Así mismo, el análisis de cada área permite destacar los factores de mayor prevalencia a fin de priorizar los niveles de intervención. (p.38)

Por su parte, Charria, Sarsosa, y Arenas (2011) refieren en cuanto a este instrumento no se reconocen evidencias respecto a su validación e indicadores de confiablidad. Pero ha tenido utilización para varias investigaciones que tratan de la identificación de factores de riesgo psicosocial, particularmente en la población de docentes universitarios.

Teniendo en cuenta lo anterior, de este modelo se concluyen los siguientes elementos: hasta el momento no existen evidencias del diseño del instrumento de acuerdo con la contextualización de las características de la población colombiana, que permita dar cuenta del reconocimiento de los factores de riesgo psicosociales según su exposición en los escenarios de los trabajadores de este país, por otro lado, tampoco aborda todos los elementos exigidos por la Resolución 2646 de 2008 expedida por el Ministerio de la Protección Social, la cual regula los factores de Riesgos Psicosociales Laborales en Colombia. Esto porque el instrumento fue creado antes de la expedición de esta ley. Sin embargo, a la fecha no se evidencian modificaciones del instrumento en aras de adaptarlo a la normatividad vigente.

\section{La batería para la evaluación de factores de riesgo psicosocial (intralaboral y extralaboral) del Ministerio de la Protección Social}

En Colombia, a partir de la expedición de la Resolución 2646 del año 2008, se desarrolló una batería para la medición del factor de riesgo psicosocial laboral, esta batería fue diseñada por el Ministerio de la Protección Social (2010) el cual refiere que la batería posee un grupo de herramientas validadas y confiables que pueden emplear las empresas y los líderes del proceso de Salud Ocupacional, para la identificación de los factores de riesgos psicosociales laborales a los cuales se encuentran expuestos los trabajadores de diversas actividades económicas y ocupacionales. Los diferentes instrumentos que componen la batería del Ministerio de la Protección Social, se muestran en la Tabla 2. 


\section{Tabla 2}

Instrumentos de la batería para la evaluación de factores de riesgo psicosocial (intralaboral y extralaboral) del Ministerio de la Protección Social

\begin{tabular}{|c|c|c|}
\hline No. & Instrumento & Autor \\
\hline 1 & $\begin{array}{l}\text { Ficha de datos generales (Información socio-demográfica e } \\
\text { información ocupacional del trabajador). }\end{array}$ & \multirow{7}{*}{$\begin{array}{l}\text { Ministerio de la } \\
\text { Protección Social }\end{array}$} \\
\hline 2 & $\begin{array}{l}\text { Cuestionario de factores de riesgo psicosocial intralaboral } \\
\text { (forma A) }\end{array}$ & \\
\hline 3 & $\begin{array}{l}\text { Cuestionario de factores de riesgo psicosocial intralaboral } \\
\text { (forma B) }\end{array}$ & \\
\hline 4 & Cuestionario de factores de riesgo psicosocial extralaboral & \\
\hline 5 & Guía para el análisis psicosocial de los puestos de trabajo & \\
\hline 6 & $\begin{array}{l}\text { Guía de entrevistas semiestructuradas para la evaluación de } \\
\text { factores de riesgo psicosocial intralaboral }\end{array}$ & \\
\hline 7 & $\begin{array}{l}\text { Guía de grupos focales para la evaluación de factores de } \\
\text { riesgo psicosocial intralaboral }\end{array}$ & \\
\hline 8 & $\begin{array}{l}\text { Cuestionario para la evaluación del estrés (Villalobos 1996, } \\
2005 \text { y 2010) }\end{array}$ & Villalobos \\
\hline
\end{tabular}

Fuente: Modificado por los autores, considerando: (Ministerio de la Protección Social - Ficha Técnica, 2010, p.17).

Hay que tener en cuenta que el propósito de esta batería es reconocer y evaluar los factores de riesgo psicosocial intra y extralaborales en una población trabajadora que se encuentre activa (Ministerio de la Protección Social, 2010). Con respecto al modelo de análisis de los factores psicosociales, esta batería tuvo como referencia la definición de factores psicosociales expresada en la Resolución 2646 de 2008 para la construcción de sus instrumentos (Ministerio de la Protección Social, 2010), dado que en esta normatividad se expresan tres tipos de condiciones para estos factores: condiciones intralaborales, condiciones extralaborales y condiciones individuales.

Cabe destacar que la aplicación de la batería permite obtener información significativa de los empleados, tales como datos socio-demográficos y ocupacionales, así como determinar la existencia o ausencia de factores de riesgo psicosocial intra y extralaborales. Además, revela el grado de riesgo en cinco niveles: sin riesgo o riesgo despreciable, riesgo bajo, riesgo medio, riesgo alto y riesgo muy alto (Ministerio de la Protección Social, 2010).

Esta batería fue llevada a un proceso de validación de contenido donde participaron expertos, utilizando instrumentos como las entrevistas cognitivas que facilitaron la mejora de los instrumentos hacia una prueba piloto, que posteriormente conllevó a la realización de las mejoras necesarias de validación y normalización de los cuestionarios 
para la aplicación a una muestra de trabajadores afiliados al Sistema General de Riesgos Profesionales en Colombia (universo de 6’796.459 trabajadores en octubre de 2009), cuya muestra final fue de 2.360 trabajadores que respondieron el cuestionario (Ministerio de la Protección Social, 2010).

De acuerdo con lo anterior, de este modelo se concluyen los siguientes elementos: su diseño tiene en cuenta la contextualización de las características de la población trabajadora y de las empresas colombianas, respecto a los factores psicosociales a los cuales se encuentran expuestos los trabajadores de este país, también reconoce las condiciones intra, extralaborales e individuales del trabajador, tal como lo exige la Resolución 2646 de 2008 expedida por el Ministerio de la Protección Social, la cual regula los factores de Riesgos Psicosociales Laborales en Colombia. Para finalizar, cuenta con una herramienta -softwareque permite la obtención de los resultados, y su uso no tiene costo alguno para la empresa que desee utilizarla.

\section{A modo de reflexión}

Después de la revisión conceptual respecto al factor de riesgo psicosocial laboral, se identifica que no se presenta unificación en su definición, no obstante, los conceptos analizados concuerdan en tres elementos primordiales; el primero es la condición interna de la organización; con lo cual se hace referencia a las características del trabajo, la infraestructura del entorno laboral, su dinámica relacional, el contenido de las tareas y su frecuencia, y cómo estas se encuentran organizadas para el desarrollo diario de la actividad laboral, tales situaciones presentan una influencia en la salud (física, psíquica y social) del trabajador; el segundo lo constituyen las condiciones externas del trabajo, las cuales hacen referencia al ambiente en el que se desarrolla el individuo, por ejemplo su contexto familiar, social y económico, y aquellas características de estos ambientes, que también generan una influencia en la salud (física, psíquica y social) del trabajador; y tercero es la condición individual del trabajador, entendida como las características propias del sujeto que le permiten percibir y afrontar las situaciones generadoras de sus condiciones intralaborales y extralaborales.

Dentro del estudio de los factores de riesgo psicosocial laboral, la Resolución 2646 (2008) refiere que en el contexto laboral se presentan cargas físicas, mentales o psíquicas, y estas se encuentran potenciadas y/o sinergizadas por las condiciones extralaborales y los factores individuales. Esto hace necesario, que siempre sean valoradas.

Los elementos comunes que existen en los conceptos, independientemente de los autores, permiten reconocer que los factores de riesgo psicosocial laboral, son generados por las organizaciones, se encuentran determinados por el contenido y las condiciones del trabajo, la realización de tareas, las interacciones en las relaciones laborales, sin dejar de lado su medio ambiente, así como también los elementos que incluyen la satisfacción en el trabajo, el clima, la cultura de la organización, todo un entorno laboral, y el entorno fuera de la organización donde se resaltan aspectos del individuo que permitan dar cuenta de sus capacidades de adaptación a este contexto y donde sus expectativas juegan un rol 
importante para enfrentar estos riesgos, que al presentarse afectación negativa conducen al estrés laboral y a otros problemas que vinculan el bienestar, la salud (física, psíquica y social) y la seguridad del trabajador.

En particular, cuando se estudian los factores de riesgo psicosocial laboral en las organizaciones, Abello, y Lozano (2013) refieren que estos factores no deben pensarse como componentes adicionales de la seguridad y la calidad de vida del trabajador, por lo que son factores reales, dado que se viven día a día y posiblemente cada vez lo sean más de acuerdo con la creciente repercusión de los factores organizacionales en la distribución del trabajo y de las empresas. No se puede dejar de lado que estos factores de riesgo llegan a ser los generadores tanto de accidentes laborales como de enfermedades ocupacionales y los altos índices de ausentismo que se vinculan a la salud de los trabajadores.

El objetivo de las herramientas de medición de los factores de riesgo psicosocial laboral es identificar y evaluar estos factores de riesgo en la población trabajadora según lo exigido por la Resolución 2646 del año 2008, emitida por el Ministerio de la Protección Social colombiano. Por lo tanto, Salanova, y Llorrens (2009) estima que la intervención inicia con la evaluación de los factores psicosociales y teniendo en cuenta los resultados obtenidos se determinan las intervenciones.

En este orden de ideas, la realización del diagnóstico de los factores psicosociales laborales, permite identificar las condiciones a las que se hallan expuestos los trabajadores, que puedan afectar su bienestar y salud (física, psíquica y social). Según Chiavenato (2000), para la comprensión de las conductas de las personas se hace necesario comprender que viven y se comportan en un escenario psicológico, y de acuerdo con esto, buscan reducir las discrepancias con el ambiente. Siendo así, tal identificación permitirá determinar las acciones de una gestión del cambio de las empresas y sus condiciones laborales, acordes con las necesidades de los trabajadores, enfocadas a mitigar y eliminar estos factores de riesgo.

De acuerdo con lo anterior, autores como López (2008) consideran que el comportamiento se encuentra relacionado con acontecimientos del ambiente de forma sistemática y previsible, de esta forma, los estudios realizados entre la conducta y el ambiente conllevarían a predecir y controlar la conducta en el trabajo. Por lo tanto, los cambios empresariales en beneficio de los trabajadores, pueden denominarse como protectores psicosociales, autores como Londoño, Sanín, \& Valencia, (2010) señalan que los protectores psicosociales, son una condición psicosocial existente en el entorno laboral del trabajador o de su colectividad, que permite minimizar o eliminar los factores de riesgo psicosocial y estos pueden afectar positivamente su salud, bienestar, desempeño y desarrollo personal.

Cabe destacar lo manifestado por Torres (2008): los esfuerzos direccionados hacia el control del estrés son más necesarios hoy en día, teniendo en cuenta los cambios generados a través del tiempo y en la actualidad en el tipo de trabajo y sus condiciones. Sin dejar de lado que Hermosa y Perilla (2015) consideran que el adoptar medidas para contrarrestar los efectos del estrés en el trabajo conlleva a beneficios para las empresas y los trabajadores. 
Al respecto, Gómez (2007) en primera instancia estima que se debe convencer a los directivos y empleados de la importancia y necesidad de implementar los programas de medición del factor de riesgo psicosocial en las empresas, con énfasis en los costos de los accidentes de trabajo y de las enfermedades ocupacionales, seguidamente, dejar en claro que las acciones del programa deben priorizarse de acuerdo con las necesidades de los trabajadores y por último, ser explícitos en la evaluación continua de programa, sin dejar de lado el objetivo principal de proteger y promover la salud de los trabajadores. Por lo tanto, Henao (2010) revela que las actuaciones llevadas a cabo en la salud ocupacional son preventivas, y tienen un enfoque de protección, formación, prevención y sobre todo, de control de factores de riesgo ambiental, con el propósito de impedir que se presenten accidentes de trabajo y se generen enfermedades profesionales en los trabajadores.

Así mismo, en lo que respecta a las dos herramientas de medición de los factores de riesgo psicosocial laboral que se plantean en el cuerpo de este artículo, solo la batería del Ministerio de la Protección Social contiene en su estructura las variables que permiten medir las condiciones intralaboral, extralaboral y las condiciones individuales, encontrándose validada en las empresas colombianas y sus diferentes sectores económicos, por lo cual reconoce las características de los trabajadores colombianos frente a la exposición de estos factores de riesgo, lo cual conlleva al cumplimiento de la Resolución 2646 del 2008, expedida por el Ministerio de la Protección Social de Colombia.

Cabe destacar que la Herramienta de Bocanument y Barján no cumple con los lineamientos exigidos por parte de la resolución 2646 de 2008 expedida por el Ministerio de la Protección Social, dado que su diseño fue en años anteriores a la expedición de esta norma, y en la actualidad no se evidencian ajustes del instrumento para su adaptación respecto a lo exigido de ley. Hay que hacer notar, que mediante respuesta a radicado 79800 Derecho de petición Evaluación Factores Psicosociales, el Ministerio de Trabajo (2015) refiere que las empresas pueden utilizar otras herramientas de evaluación del factor de riesgo psicosocial, siempre y cuando estas cumplan con lo determinado en la resolución 2646 de 2008.

Por lo tanto, la batería del Ministerio de Protección Social, genera un significativo aporte a las empresas en el desarrollo de la prevención de los riesgos, las enfermedades de origen laboral, y contribuye hacia los cambios organizacionales, en beneficio tanto de empresarios como de empleados en aras del Sistema de Gestión para la Seguridad y la Salud en el trabajo. De esta forma, autores como López (2015) afirman que el sistema de gestión de seguridad y salud en el trabajo es una herramienta significativa para garantizar el compromiso de quienes se benefician del trabajo. Para González, Giachero, y Delgado (2012) los riesgos psicosociales son los nuevos riesgos emergentes y su origen se encuentra en las condiciones de trabajo deficientes, particularmente en su organización.

Finalmente, el diagnóstico de los factores de riesgo psicosocial laboral, se hace importante debido a la necesidad de prevención y de implementación de acciones correctivas de las condiciones intra, extralaborales e individuales, que afectan de manera negativa la 
salud de los trabajadores. Es decir, tal como Ramos, Crespo, Jáuregui, \& Cassola (2008) manifiestan que la importancia del estudio de los riesgos psicológicos radica en tener en cuenta los factores que afectan al trabajador, tanto los físicos como los socio-psicológicos. Autores como Maestre (2008) aseguran que las condiciones desfavorables en el contexto laboral, pueden dar origen a la presencia de conductas y actitudes inadecuadas en este mismo contexto, y pueden traer consecuencias que lesionarán la salud y el bienestar de los trabajadores.

Por lo anterior, Daza, Linares, y Martínez (2004) asumen que al no tomar medidas para prevenir y corregir los factores de riesgo psicosociales que son posibles generadores de estrés, existiría afectación en la salud mental, lo cual conlleva alteraciones en la salud del trabajador, y en su productividad. Se puede señalar, que los empleadores cuya representación es la alta gerencia, se encuentran en la obligación de implementar programas de vigilancia epidemiológica del factor de riesgo psicosocial laboral, donde el Ministerio de Trabajo (2015) señala que las empresas no pueden delegar la responsabilidad de la medición de los factores de riesgo psicosociales laborales, a las administradoras de riesgos laborales.

Además, el diseño debe permitir la participación de los trabajadores como actores involucrados debido a su exposición continua a las condiciones del contexto laboral, el contenido de las tareas y del ambiente de trabajo, entre otros elementos que conceptualizan este factor de riesgo que actualmente afecta negativamente su salud. Ahora bien, según Zapata y Rodríguez (2008), se han desarrollado estilos de liderazgo democráticos, que suponen tener en cuenta a los trabajadores, subordinados y/o seguidores para tomar decisiones en situaciones necesarias y de contingencia para la misma empresa.

Tal liderazgo democrático, permitirá orientar adecuadamente los planes, teniendo en cuenta la gestión de conocimiento de cada trabajador, para el desarrollo de estrategias acordes a sus necesidades y enmarcando las prioridades de intervención, situación que conllevará hacia un mejor bienestar y prevención de accidentes de trabajo y enfermedades de origen laboral. Por lo tanto, Gil (2012) refiere que existen estudios los cuales reconocen el lugar de trabajo, como un lugar privilegiado para el desarrollo de actuaciones en función de la prevención de los riesgos psicológicos y promover la salud mental. Sin dejar de lado que Domínguez (1997) refiere que la ética empresarial está constituida por unos valores que deben ser incuestionables, lo cual hace que las sociedades crezcan sin violencia porque tienen equidad y justicia.

Esto lleva a reconocer que las actuaciones realizadas por las empresas en aras de la prevención y salud de los trabajadores, tiene efectos para toda una sociedad, por lo cual González, y Contreras (2016) refieren que el bienestar de los trabajadores y de la sociedad en general, presentan una relación directa e indiscutible. Por lo cual, todas las situaciones que generen efectos en las condiciones de trabajo, tendrán incidencia en la calidad de vida de los habitantes de un país. 


\section{Futuras investigaciones}

Se hace necesario, posteriormente llevar a cabo investigaciones sobre los factores de riesgos psicosociales, que permitan obtener mayor información sobre estos tipos de factores de riesgos en Colombia, particularmente en los siguientes ámbitos:

- Eficacia de la batería del Ministerio de la Protección Social, como instrumento diseñado, publicado, reconocido y validado estadísticamente hasta el momento en Colombia para la medición del factor de riesgo psicosocial laboral.

- Impacto de la implementación del Sistema de Vigilancia Epidemiológico del factor de riesgo psicosocial laboral en Colombia, y sus efectos en la salud laboral de los trabajadores.

- Estudios de las consecuencias de las enfermedades laborales en Colombia.

- Estudios en las empresas colombianas, con el fin de identificar la existencia de riesgos psicosociales de acuerdo con el sector.

- Identificación de otros instrumentos diseñados para la medición del factor de riesgo psicosocial laboral, que cumplan los lineamientos brindados por la resolución 2646 de 2008 expedida por el Ministerio de la Protección Social.

\section{Conflicto de intereses}

Los autores declaran no tener ningún conflicto de intereses.

\section{Referencias Bibliográficas}

1. Abello, B., y Lozano, T. (2013). Importancia de los factores de riesgo psicosocial y clima organizacional en el ámbito laboral. Universidad del Rosario, Bogotá, Colombia.

2. Álvarez, F. T., Londoño, M. E. L., Posada, A. S., \& Jáuregui, M. V. (2016). Modelo analítico de factores psicosociales en contextos laborales. Revista Interamericana de Psicología Ocupacional, 29(2), 95-137.

3. Álvarez, F., Conti, L., Valderrama, F., Moreno, O. y Jiménez, I. (2007). Salud Ocupacional. Bogotá, Colombia: Ecoe.

4. Arias Gallegos, W. L. (2012). Estrés laboral en trabajadores desde el enfoque de los sucesos vitales. Revista Cubana de Salud Pública, 38(4), 525-535.

5. Bestratén, M. (2015). Gestión de la prevención en un marco de excelencia. Barcelona: España: Universitat Oberta de Catalunya.

6. Blanch, J., Espuny, M., Gala, C., y Artiles, A. (2003). Teoría de las relaciones laborales: Desafíos. Barcelona, España: UOC

7. Boada, J, y Ficapal, P. (2012). Salud y Trabajo los nuevos y emergentes riesgos psicosociales. Universidad Oberta de Catalunya, Barcelona, España.

8. Bocanument, G. (1994). Naturaleza, origen clasificación y evaluación de los factores de riesgo sico-sociales del trabajo. Revista administración de personal. 16 (52), 31-49.

9. Buceta, J., Bueno, A., y Mas, B. (2001). Intervención Psicológica y Salud: Control del estrés y conductas de riesgo. Madrid, España: Dykinson.

10. Cardona, Á. E. M. (2006). El empresario de sí mismo: una visión desde la economía. Revista Ciencias Estratégicas, 14(15), 67-82. 
11. Carvajal, O., y Dávila, C. (2013). Mobbing o acoso laboral. Revisión del tema en Colombia. Facultad de Ciencias de la Administración. Universidad del Valle. Vol.29 (49), 100.

12. Charria O., V. H., Sarsosa, P., K. V., \& Arenas O., F. (2011). Factores de riesgo psicosocial laboral: métodos e instrumentos de evaluación. Revista Facultad Nacional De Salud Pública, 29(4), 380-391

13. Chiavenato, I. (2000). Administración de Recursos Humanos. Colombia: Editorial McGraw-Hill

14. Creus, A. (2011). Técnicas para la prevención de riesgos laborales. Barcelona, España: Marcombo

15. Comité Mixto O.I.T. y O.M.S. 1984. Factores psicosociales en el trabajo: Naturaleza, incidencia y prevención. Ginebra: OIT/OMS.

16. Congreso de Colombia (2006). Ley 1010 de 2006.

17. Daza González, N. E., Linares Chinchilla, Y., \& Martínez, J. (2012). Factores de riesgos psicosociales generadores de estrés laboral en Parmalat Colombia Ltda. (Área industrial y administrativa de la planta Bogotá).

18. Díaz, R., Echeverri, A., Ramírez, G., y Ramírez, G. (2010) Diagnóstico del riesgo psicosocial, en trabajadores del área de la salud (Tesis de postgrado) Universidad CES, Medellín, Colombia.

19. Domínguez, J. I. (2012). Impacto económico de los accidentes de trabajo. Revista Universidad Eafit, 33(107), 89-96.

20. Gamero Merino, C. (2013). Manual de riesgos psicosociales en el trabajo: teoría y práctica. Revista de la Asociación Española de Neuropsiquiatría, 33(117), 209-211.

21. González, K. A., \& Contreras, M. Y. (2016). Costos de los Accidentes Laborales: Colombia, 2009-2012. Ciencias Psicológicas, 10 (1), 31-41.

22. Gómez, I. C. (2007). Salud Laboral: Una revisión a la luz de las nuevas condiciones del trabajo. Universitas Psychologica. 6(1), 105-113.

23. Gómez, Ortiz, V. \& Moreno, L. (2010). Factores psicosociales del trabajo (demandacontrol y desbalance esfuerzo-recompensa), salud mental y tensión arterial: un estudio con maestros escolares en Bogotá, Colombia. Universitas Psychologica, 9 (2), 393-407.

24. Henao, Robledo, F. (2010). Salud Ocupacional: conceptos básicos. Bogotá: Ecoe Ediciones.

25. Henao, F. (2013). Seguridad y salud en el trabajo: conceptos básicos. Bogotá, Colombia: Ecoe Ediciones.

26. Hermosa R., A.M., \& Perilla T., L.E. (2015). Retos investigativos en psicología de la salud ocupacional: el estrés laboral. Revista facultad Nacional De Salud Pública, 33(2), 252-261.

27. López, J., María. (1996). Psicología Ocupacional. Colombia. Universidad del Quindío.

28. López, M., Luis (2008). Intervención Psicológica en la Empresa. España. Editorial Pirámide.

29. López Cortés, O. A. (2015). El riesgo psicosocial en la legislación colombiana: el gobierno de lo imprevisible. Diálogos de Saberes, (43), 57-72.

30. Mateo, P. (2013). Control del Estrés Laboral. Madrid, España: Fundación Confemetal.

31. Maestre, D. (2008). Ergonomía y psicología. Madrid, España: Fundación Confemetal.

32. Ministerio de trabajo y seguridad social, MTySS (1984). Decreto 614 de 1984. 
33. Ministerio de Gobierno de la república de Colombia (1994). Decreto 1295 de 1994. http://www.alcaldiabogota.gov.co/sisjur/normas/Norma1.jsp?i=2629

34. Ministerio de Trabajo y Seguridad Social, MTySS (2008). Resolución 2646 de 2008.

35. Ministerio de la Protección Social (2009). Decreto 2566 de 2009.

36. Ministerio de la Protección Social (2010). Batería de instrumentos para la evaluación de factores de riesgo psicosocial.

37. Ministerio de Trabajo y Seguridad Social, MTySS (2012). Ley 1562 de 2012.

38. Ministerio de Trabajo y Seguridad Social, MTySS (2014). Decreto 1477 de 2014.

39. Ministerio del Trabajo (2015). Decreto número 1072 de 2015.

40. Ministerio del Trabajo (2015). Rad.79800 Derecho de Petición Evaluación Factores Psicosociales.

41. Moreno, B., y Garrosa, E. (2013). Salud laboral. Riesgos laborales psicosociales y bienestar laboral. Madrid, España: Pirámide.

42. Pedro R., G. (2012). Riesgos psicosociales en el trabajo y salud ocupacional / Psychosocial risks at work and occupational health. Revista Peruana de Medicina Experimental y Salud Pública, (2), 237.

43. Peiró, J. (2009). Estrés laboral y riesgos psicosociales: Investigaciones recientes para su análisis y prevención. Valencia, España: Universitat de Valéncia.

44. Pontificia Universidad Javeriana (2007). Protocolo para la determinación del origen de las patologías derivadas del estrés. Bogotá: Universidad Javeriana, Subcentro de Seguridad Social y Riesgos Profesionales.

45. Rivas, P. (2009) La prevención de los riesgos laborales de carácter psicosocial. P.3 Granada: Editorial Comares.

46. Ramos, V. R., Crespo, J. M., Jáuregui, L. C., \& Cassola, G.A. (2008). La importancia de los riesgos psicológicos: identificación. Gestión Práctica de Riesgos Laborales. (53), 46-51.

47. Salanova, M., \& Llorens, S. (2009). Presente y futuro de la intervención profesional en factores psicosociales. Gestión Práctica De Riesgos Laborales, (59), 20-23.

48. Sauter SL, Murphy LR, Hurrel JJ, Levi L. (2001) Factores psicosociales y de organización. Capítulo 34. En: Enciclopedia de salud y seguridad en el trabajo. $3^{\circ}$ ed. España: Organización Internacional del Trabajo y Ministerio de Trabajo y Asuntos Sociales.

49. Tomasina, F. (2012). Los problemas en el mundo del trabajo y su impacto en salud. Crisis financiera actual. Revista de Salud Pública, 14(1s), 56-67.

50. Torres, Idier (2008). Control del Estrés Laboral. En Pando, Román y Acosta (Comps.). Factores Psicosociales de riesgo de Trabajo en la Empresa (pp.104-119). La Habana, Cuba. Red Iberoamericana de Riesgos Psicosociales Laborales RIPSOL.

51. Varillas, Walter. (2009). Los trabajadores y la salud mental. El reto de la prevención y el tratamiento en un mundo enfermo. La Habana, Cuba: Ciencias Médicas.

52. Vieco Gómez, G.F., \& Llanos, R.A. (2014). FACTORES PSICOSOCIALES DE ORIGEN LABORAL, ESTRÉS Y MORBILIDAD EN EL MUNDO. Psicología Desde el Caribe, 31(2), 354-385.

53. Zapata, A., y Rodríguez, A. (2008). Cultura Organizacional. Colombia: Editorial Universidad del Valle. 